\title{
A finite element method for the resolution of the Reduced Navier-Stokes/Prandtl equations
}

\author{
Gabriel R. Barrenechea and Franz Chouly \\ Departamento de Ingeniería Matemática, Universidad de Concepción, Casilla 160-C, Concepción, Chile. \\ gbarrene@ing-mat.udec.cl, fchouly@ing-mat.udec.cl.
}

\begin{abstract}
A finite element method to solve the bidimensional Reduced Navier-Stokes Prandtl (RNS/P) equations is described. These equations are an asymptotical simplification of the full Navier-Stokes equations, obtained when one dimension of the domain is of one order smaller than the others. These are therefore of particular interest to describe flows in channels or pipes of small diameter. A low order finite element discretization, based on a piecewise constant approximation of the pressure, is proposed and analyzed. Numerical experiments which consist in fluid flow simulations within a constricted pipe are provided. Comparisons with Navier-Stokes simulations allow to evaluate the performance of prediction of the finite element method, and of the model itself.
\end{abstract}

Keywords : incompressible flow, RNS/P equations, finite elements.

\section{Introduction}

For some kind of flow problems in which the domain is a long tube of small diameter, such as flows in a Venturi pipe [20] or some biological flows (stenosis [12], or human upper airways $[11,21,5])$, it is reasonable to simplify the full Navier-Stokes equations assuming that the ratio of the characteristic lengths is infinitely small. One possible simplification, proposed in [12] based on asymptotic expansions, are the Reduced-Navier Stokes/Prandtl (RNS/P) equations. The interest being a better adequation with the relevant scalings of the fluid flow problem.

For these equations, a numerical method based on finite differences has been proposed and tested in [11]. Even if the proposed method is cheap and adaptable to some different geometries, it has some important drawbacks. Among them, we can quote:

- first, it lacks of robustness. Specifically, for some categories of geometries, such as constrictions, numerical problems occur after the separation of the flow. This is mostly due to recirculation effects, which cannot be easily taken into account in the finite differences framework. The standard method is to use the "FLARE" approximation [15] which consists in removing the $u \partial_{x} u$ term when the longitudinal velocity is negative [12]. However, this is an ad-hoc approximation and does not ensure a correct computation in the whole domain.

- Some care has to be taken when adapting a finite differences scheme to complicated geometries.

- If we are interested in fluid-structure interaction problems (such as in the upper airways $[6]$ ), then if the motion of the structure is solved using the finite element method, as it is usually done, the transmission of the forces at the interface can not be done in a simple and natural way (see [6] for the details). 
As a result of the previous considerations, in this work we are interested in the first steps towards a finite element method for the resolution of the RNS/P equations, which should avoid some of these disadvantages.

The plan of the paper is as follows. First, the complete boundary value problem is given in Section 2. The finite element method is described and analyzed in Section 3; in particular, since finite elements of common use for the Navier-Stokes equations - namely Taylor-Hood element [22] and the Mini element [8] - do not provide a correct approximation, we use a specific finite element, originally proposed in [18] for the Stokes equation. It is shown that with this method, the discrete problem admits a solution. Numerical experiments, presented in Section 4, have been carried out to confirm the analysis, and to test the precision of the method through comparison with Navier-Stokes simulations, taken as a reference. Finally some concluding remarks and perspectives are drawn.

\section{The boundary value problem}

The Reduced Navier-Stokes/Prandtl (RNS/P) equations are derived from the Navier-Stokes (1) equation. For the sake of simplicity, one can assume a steady, incompressible, laminar and bidimensional flow:

$$
\left\{\begin{aligned}
(\boldsymbol{u} \cdot \nabla) \boldsymbol{u} & =-\frac{1}{\rho} \nabla p+\nu \Delta \boldsymbol{u}+\boldsymbol{g}, \\
\nabla \cdot \boldsymbol{u} & =0,
\end{aligned}\right.
$$

where $\boldsymbol{u}$ is the velocity, $p$ is the pressure, $\rho$ is the density, $\nu$ is the kinematic viscosity and $\boldsymbol{g}$ is the external force field; $\boldsymbol{g}$ is in a great amount of applications the gravity field but may also stand for any kind of other external influence (e.g. a magnetic field). To derive the RNS/P equations, we need two assumptions, namely:

1. if we note $D_{2}$ the transversal dimension of the domain and $D_{1}$ the longitudinal dimension, then $D_{1} / D_{2} \gg 1$ (Fig. 1).

2. if the Reynolds number is defined as $\operatorname{Re}=U_{0} D_{2} / \nu$, where $U_{0}$ stands for the maximal velocity at the entry, then $\operatorname{Re} \gg 1$.

Then, the Navier-Stokes equation (1) can be simplified in order to obtain Reduced NavierStokes / Prandtl (RNS/P) equations (see [12] for the derivation):

$$
\left\{\begin{aligned}
u_{1} \partial_{x_{1}} u_{1}+u_{2} \partial_{x_{2}} u_{1} & =-\frac{1}{\rho} \partial_{x_{1}} p+\nu \partial_{x_{2}^{2}}^{2} u_{1}+g_{1}, \\
\partial_{x_{2}} p & =0 \\
\partial_{x_{1}} u_{1}+\partial_{x_{2}} u_{2} & =0
\end{aligned}\right.
$$

Here, $\left(u_{1}, u_{2}\right)$ are respectively the longitudinal and the transversal components of the velocity $\boldsymbol{u}$, and $g_{1}$ is the longitudinal component of the external force field $\boldsymbol{g}$. In the case of a gravity field, it means of course that the gravity is taken into account only if the duct is not horizontal. Boundary conditions consist of no slip on the lower and upper walls as well as an inlet flow at the entrance. The exit of the domain is considered as free. As a result, the RNS/P equations are the Prandtl boundary layer equations [4] with two major differences: 


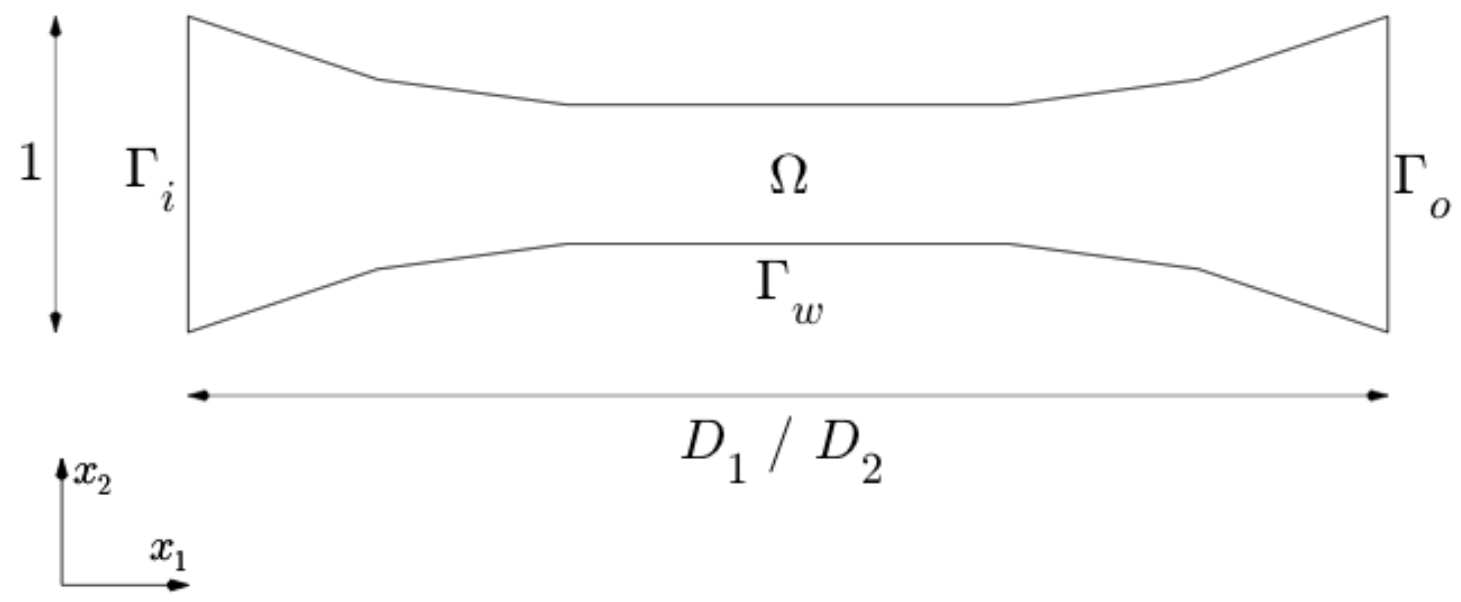

Fig. 1. The domain $\Omega$ for the resolution of the RNS/P equations, with the notations for the different parts of the boundary. Note that $x_{1}$ and $x_{2}$ are non-dimensional coordinates.

1. the domain in the RNS/P formulation is bounded in the transverse direction and there is no more fitting at the infinity with the inviscid flow;

2. the pressure distribution in the domain is an unknown.

Let us consider $\Omega$ which is a polygonal domain in $\mathbb{R}^{2}$ with boundary $\partial \Omega ; \Gamma_{i} \subset \partial \Omega$ is the entry (inlet flow), $\Gamma_{w} \subset \partial \Omega$ is the rigid wall (with no-slip boundary conditions) and $\Gamma_{o} \subset \partial \Omega$ is the exit (outlet flow) (Fig. 1). We give now the full boundary value problem that aims to be solved, in a non-dimensional form:

$$
\left\{\begin{aligned}
u_{1} \partial_{x_{1}} u_{1}+u_{2} \partial_{x_{2}} u_{1}+\partial_{x_{1}} p-\frac{1}{\operatorname{Re}} \partial_{x_{2}^{2}}^{2} u_{1}=g_{1} & \text { in } \Omega, \\
\partial_{x_{2}}=0 & \text { in } \Omega, \\
\partial_{x_{1}} u_{1}+\partial_{x_{2}} u_{2}=0 & \text { in } \Omega, \\
u_{1}=u_{1}^{0} & \text { on } \Gamma_{i}, \\
u_{1}=0 & \text { on } \Gamma_{w}, \\
u_{2} n_{2}=0 & \text { on } \Gamma_{w} .
\end{aligned}\right.
$$

The velocity profile $u_{1}^{0}$ at the entry may be arbitrary, usually a flat profile or a Poiseuille profile.

\section{The finite element method}

As in the case of Stokes or Navier-Stokes equation, a finite element method can be proposed to solve the RNS/P equations. Moreover, some of the techniques already known to obtain a discrete approximation and to analyze it can be adapted to this case. Nevertheless, as in the case of the primitive equations of the ocean, in which the pressure is also constant in one direction of the space, finite elements such as Taylor-Hood or Mini element are inappropriate for discretization [1]. Hence, we propose a discretization using an element which was first studied 
in [18].

First, in Section 3.1, we give the weak formulation of the boundary value problem (3). Then, in Section 3.2, we introduce the finite element spaces which are used in the discrete weak formulation. A brief analysis of the discrete problem is carried out in Section 3.3. Finally, the complete algorithm of numerical resolution is detailed in Section 3.4.

\subsection{Weak formulation}

To avoid technical difficulties, the problem (3) is rewritten with homogeneous Dirichlet boundary conditions on $\partial \Omega\left(\Gamma_{w}=\partial \Omega, \Gamma_{i}=\emptyset, \Gamma_{o}=\emptyset\right)$. Let us first present some notations. By $\mathcal{L}^{2}(\Omega)$ we denote the space of square integrable scalar functions on $\Omega,(\cdot, \cdot)_{\Omega}$ stands for the inner product in $\mathcal{L}^{2}(\Omega)$ (in $\mathcal{L}^{2}(\Omega)^{2}$ or in $\mathcal{L}^{2}(\Omega)^{2 \times 2}$, if necessary); $\|.\|_{0, \Omega}$ stands for the norm in $\mathcal{L}^{2}(\Omega)$ associated to $(\cdot, \cdot)_{\Omega}$. $\mathcal{L}_{0}^{2}(\Omega)$ is the subspace of functions in $\mathcal{L}^{2}(\Omega)$ with zero mean value on $\Omega$. $\mathcal{H}^{1}(\Omega)$ is the space of square integrable scalar functions on $\Omega$, with square integrable first derivatives. In the sequel, we will need the following space

$$
\mathcal{H}^{1}\left(\partial_{x_{2}}, \Omega\right)=\left\{v \in \mathcal{L}^{2}(\Omega) \mid \partial_{x_{2}} v \in \mathcal{L}^{2}(\Omega)\right\} .
$$

$\mathcal{H}_{0}^{1}(\Omega)$ stands for the closed subspace of $\mathcal{H}^{1}(\Omega)$ with vanishing trace on $\partial \Omega$. Similarly, we note $\mathcal{H}_{0}^{1}\left(\partial_{x_{2}}, \Omega\right)$ the following space:

$$
\mathcal{H}_{0}^{1}\left(\partial_{x_{2}}, \Omega\right)=\left\{v \in \mathcal{H}^{1}\left(\partial_{x_{2}}, \Omega\right)\left|v n_{2}\right|_{\partial \Omega}=0\right\} .
$$

$\boldsymbol{H}^{\mathbf{1}}(\Omega), \boldsymbol{H}(\Omega)$ and $\boldsymbol{H}_{\mathbf{0}}(\Omega)$ are the following spaces of vector-valued functions:

$$
\begin{aligned}
& \boldsymbol{H}^{1}(\Omega)=\mathcal{H}^{1}(\Omega) \times \mathcal{H}^{1}(\Omega), \\
& \boldsymbol{H}(\Omega)=\mathcal{H}^{1}(\Omega) \times \mathcal{H}^{1}\left(\partial_{x_{2}}, \Omega\right), \\
& \boldsymbol{H}_{\mathbf{0}}(\Omega)=\mathcal{H}_{0}^{1}(\Omega) \times \mathcal{H}_{0}^{1}\left(\partial_{x_{2}}, \Omega\right) .
\end{aligned}
$$

$\boldsymbol{H}^{\mathbf{1}}(\Omega)$ is a Hilbert space with the following scalar product:

$$
(\boldsymbol{u}, \boldsymbol{v})_{1, \Omega}=(\boldsymbol{u}, \boldsymbol{v})_{\Omega}+(\nabla \boldsymbol{u}, \nabla \boldsymbol{v})_{\Omega} .
$$

$\boldsymbol{H}(\Omega)$ is also a Hilbert space with the scalar product:

$$
(\boldsymbol{u}, \boldsymbol{v})_{\boldsymbol{H}(\Omega)}=(\boldsymbol{u}, \boldsymbol{v})_{\Omega}+\left(\nabla u_{1}, \nabla v_{1}\right)_{\Omega}+\left(\partial_{x_{2}} u_{2}, \partial_{x_{2}} v_{2}\right)_{\Omega} .
$$

This implies the following property on the norms:

$$
\forall \boldsymbol{v} \in \boldsymbol{H}^{\mathbf{1}}(\Omega),\|\boldsymbol{v}\|_{\boldsymbol{H}(\Omega)} \leq\|\boldsymbol{v}\|_{1, \Omega},
$$

with $\|\cdot\|_{1, \Omega}$ the norm on $\boldsymbol{H}^{\mathbf{1}}(\Omega)$ associated to $(\cdot, \cdot)_{1, \Omega}$. Moreover, let us note $c(\cdot, \cdot, \cdot), a_{\mathrm{Re}}(\cdot, \cdot)$, $a_{\lambda}(\cdot, \cdot)$ and $a(\cdot, \cdot)$ the continuous trilinear and bilinear forms on $\boldsymbol{H}_{\mathbf{0}}(\Omega)$ defined by:

$$
\begin{aligned}
& c \quad:(\boldsymbol{u}, \boldsymbol{v}, \boldsymbol{w}) \longmapsto\left(u_{1} \partial_{x_{1}} v_{1}+u_{2} \partial_{x_{2}} v_{1}, w_{1}\right)_{\Omega}, \\
& a_{\mathrm{Re}}:(\boldsymbol{u}, \boldsymbol{v}) \longmapsto \frac{1}{\operatorname{Re}}\left(\partial_{x_{2}} u_{1}, \partial_{x_{2}} v_{1}\right)_{\Omega}, \\
& a_{\lambda}:(\boldsymbol{u}, \boldsymbol{v}) \longmapsto(\lambda \nabla \cdot \boldsymbol{u}, \nabla \cdot \boldsymbol{v})_{\Omega}, \\
& a:(\boldsymbol{u}, \boldsymbol{v}) \longmapsto a_{\operatorname{Re}}(\boldsymbol{u}, \boldsymbol{v})+a_{\lambda}(\boldsymbol{u}, \boldsymbol{v}) \text {. }
\end{aligned}
$$


Here, $\lambda$ is a non-negative (possibly equal to zero) scalar field over $\Omega$. The least-squares term $a_{\lambda}$ can be added into the variational formulation without affecting the solution. Although irrelevant for the continuous problem, this least-squares term has an effect on the solution of the discrete problem (see Section 4 for a discussion). We also introduce the continuous bilinear form $b(\cdot, \cdot): \mathcal{L}_{0}^{2}(\Omega) \times \boldsymbol{H}_{\mathbf{0}}(\Omega) \rightarrow \mathbb{R}$ defined by:

$$
b:(p, \boldsymbol{v}) \longmapsto-(p, \nabla \cdot \boldsymbol{v})_{\Omega} .
$$

Using these forms, we present the following weak formulation for (3): Find $(\boldsymbol{u}, p) \in \boldsymbol{H}_{\mathbf{0}}(\Omega) \times$ $\mathcal{L}_{0}^{2}(\Omega)$ such that:

$$
\left\{\begin{array}{cc}
\forall \boldsymbol{v} \in \boldsymbol{H}_{\mathbf{0}}(\Omega), \quad c(\boldsymbol{u}, \boldsymbol{u}, \boldsymbol{v})+a(\boldsymbol{u}, \boldsymbol{v})+b(p, \boldsymbol{v})=(\boldsymbol{g}, \boldsymbol{v})_{\Omega}, \\
\forall q \in \mathcal{L}_{0}^{2}(\Omega), & b(q, \boldsymbol{u})=0 .
\end{array}\right.
$$

Note that from the boundary value problem, we have $\boldsymbol{g}=\left(g_{1}, 0\right)$. Nevertheless, we will consider the general case $g_{2} \neq 0$ in the rest of the text. For the analysis of the problem, we introduce the following space:

$$
\boldsymbol{H}_{\boldsymbol{b}}(\Omega) \stackrel{\mathrm{DEF}}{=} \operatorname{ker} b=\left\{\boldsymbol{v} \in \boldsymbol{H}_{\mathbf{0}}(\Omega) \mid \forall q \in \mathcal{L}_{0}^{2}(\Omega), b(q, \boldsymbol{v})=0\right\}=\left\{\boldsymbol{v} \in \boldsymbol{H}_{\mathbf{0}}(\Omega) \mid \nabla \cdot \boldsymbol{v}=0\right\}
$$

where the last equality arises from the fact that $\nabla \cdot \boldsymbol{v} \in \mathcal{L}_{0}^{2}(\Omega)$. As for the Navier-Stokes and Stokes equations, we reformulate this mixed weak formulation into two dependent problems. For stability reasons (see Lemma 2 below), the convective term is transformed using the following proposition:

Proposition 1. The trilinear form c can be decomposed into a symmetric and antisymmetric part, noted $c_{\mathrm{s}}$ and $c_{\mathrm{a}}$, respectively, with the following expression for the symmetric part:

$$
\forall \boldsymbol{u}, \boldsymbol{v}, \boldsymbol{w} \in \boldsymbol{H}_{\mathbf{0}}(\Omega), c_{\mathrm{S}}(\boldsymbol{u}, \boldsymbol{v}, \boldsymbol{w})=-\frac{1}{2}\left(\nabla \cdot \boldsymbol{u}, v_{1} w_{1}\right)_{\Omega}
$$

As a result, the symmetric part vanishes on $\boldsymbol{H}_{\boldsymbol{b}}(\Omega)$ and the trilinear form is equal to its antisymmetric part:

$$
\begin{aligned}
& \forall \boldsymbol{u}, \boldsymbol{v}, \boldsymbol{w} \in \boldsymbol{H}_{\boldsymbol{b}}(\Omega), \\
& c(\boldsymbol{u}, \boldsymbol{v}, \boldsymbol{w})=c_{\mathrm{a}}(\boldsymbol{u}, \boldsymbol{v}, \boldsymbol{w})=\frac{1}{2}\left(\left(u_{1} \partial_{x_{1}} v_{1}+u_{2} \partial_{x_{2}} v_{1}, w_{1}\right)_{\Omega}-\left(u_{1} \partial_{x_{1}} w_{1}+u_{2} \partial_{x_{2}} w_{1}, v_{1}\right)_{\Omega}\right) .
\end{aligned}
$$

Proof. Using the Green theorem, we easily see that $c(\boldsymbol{u}, \boldsymbol{v}, \boldsymbol{w})=-\left(\nabla \cdot \boldsymbol{u}, v_{1} w_{1}\right)_{\Omega}-c(\boldsymbol{u}, \boldsymbol{w}, \boldsymbol{v})$. As a result, we note that:

$$
c_{\mathrm{S}}(\boldsymbol{u}, \boldsymbol{v}, \boldsymbol{w})=\frac{1}{2}(c(\boldsymbol{u}, \boldsymbol{v}, \boldsymbol{w})+c(\boldsymbol{u}, \boldsymbol{w}, \boldsymbol{v}))=-\frac{1}{2}\left(\nabla \cdot \boldsymbol{u}, v_{1} w_{1}\right)_{\Omega},
$$

which proves the first assertion. The second part of the proposition follows directly from the definition of the space $\boldsymbol{H}_{\boldsymbol{b}}(\Omega)$.

Using the last proposition we can propose the following equivalent formulation for the problem $(12)$ : 
- Find $\boldsymbol{u} \in \boldsymbol{H}_{\boldsymbol{b}}(\Omega)$ such that :

$$
\forall \boldsymbol{v} \in \boldsymbol{H}_{\boldsymbol{b}}(\Omega), \quad c_{\mathrm{a}}(\boldsymbol{u}, \boldsymbol{u}, \boldsymbol{v})+a(\boldsymbol{u}, \boldsymbol{v})=(\boldsymbol{g}, \boldsymbol{v})_{\Omega},
$$

- Find $p \in \mathcal{L}_{0}^{2}(\Omega)$ such that :

$$
\forall \boldsymbol{v} \in \boldsymbol{H}_{\mathbf{0}}(\Omega), \quad b(p, \boldsymbol{v})=(\boldsymbol{g}, \boldsymbol{v})_{\Omega}-c_{\mathrm{a}}(\boldsymbol{u}, \boldsymbol{u}, \boldsymbol{v})-a(\boldsymbol{u}, \boldsymbol{v}) .
$$

\section{$3.2 \quad$ Finite element spaces}

For the continuous problem (16)-(17), a discretization first proposed in [18] for the Stokes problem has been chosen: a discontinuous approximation of the pressure is preferred as it provides local conservation of the mass (cf. [9]). Let $\left\{\mathcal{T}_{h}\right\}_{h>0}$ be a regular family of admissible triangulations of $\Omega$ (cf. [8]). For each $K \in \mathcal{T}_{h}, h_{K}$ stands for the element diameter and $h=$ $\max _{\left(K \in \mathcal{T}_{h}\right)} h_{K}$. The following space has been chosen for the velocity field:

$$
\boldsymbol{H}_{\boldsymbol{h}}=\left(H_{h, 2} \cap \mathcal{H}_{0}^{1}(\Omega)\right) \times\left(H_{h, 1} \cap \mathcal{H}_{0}^{1}\left(\partial_{x_{2}}, \Omega\right)\right),
$$

where, for $k=1,2$ :

$$
H_{h, k}=\left\{v \in \mathcal{C}^{0}(\bar{\Omega})\left|\forall K \in \mathcal{T}_{h}, v\right|_{K} \in \mathbb{P}_{k}(K)\right\} .
$$

We also need to introduce the space $\boldsymbol{H}_{\boldsymbol{h}, \boldsymbol{b}}$, defined as follows:

$$
\boldsymbol{H}_{\boldsymbol{h}, \boldsymbol{b}}=\left\{\boldsymbol{v}_{h} \in \boldsymbol{H}_{\boldsymbol{h}} \mid \forall q_{h} \in \Pi_{h}, b\left(q_{h}, \boldsymbol{v}_{h}\right)=0\right\} .
$$

We note that $\boldsymbol{H}_{\boldsymbol{h}, \boldsymbol{b}}$ is not necessarily a subspace of $\boldsymbol{H}_{\boldsymbol{b}}(\Omega)$. The pressure is approximated using the following space:

$$
\Pi_{h}=\left\{q \in \mathcal{L}_{0}^{2}(\Omega)\left|\forall K \in \mathcal{T}_{h}, q\right|_{K} \in \mathbb{P}_{0}(K)\right\} .
$$

The finite element associated to this choice, called $\mathbb{P}_{2} / \mathbb{P}_{1} / \mathbb{P}_{0}$, is depicted in Fig. 2. Using this pair of spaces, we propose the following finite element method for (16)-(17):

- Find $\boldsymbol{u}_{h} \in \boldsymbol{H}_{\boldsymbol{h}, \boldsymbol{b}}$ such that:

$$
\forall \boldsymbol{v}_{h} \in \boldsymbol{H}_{\boldsymbol{h}, \boldsymbol{b}}, \quad c_{\mathrm{a}}\left(\boldsymbol{u}_{h}, \boldsymbol{u}_{h}, \boldsymbol{v}_{h}\right)+a\left(\boldsymbol{u}_{h}, \boldsymbol{v}_{h}\right)=\left(\boldsymbol{g}, \boldsymbol{v}_{h}\right)_{\Omega} .
$$

- Find $p_{h} \in \Pi_{h}$ such that:

$$
\forall \boldsymbol{v}_{h} \in \boldsymbol{H}_{\boldsymbol{h}}, \quad b\left(p_{h}, \boldsymbol{v}_{h}\right)=\left(\boldsymbol{g}, \boldsymbol{v}_{h}\right)_{\Omega}-c_{\mathrm{a}}\left(\boldsymbol{u}_{h}, \boldsymbol{u}_{h}, \boldsymbol{v}_{h}\right)-a\left(\boldsymbol{u}_{h}, \boldsymbol{v}_{h}\right)
$$




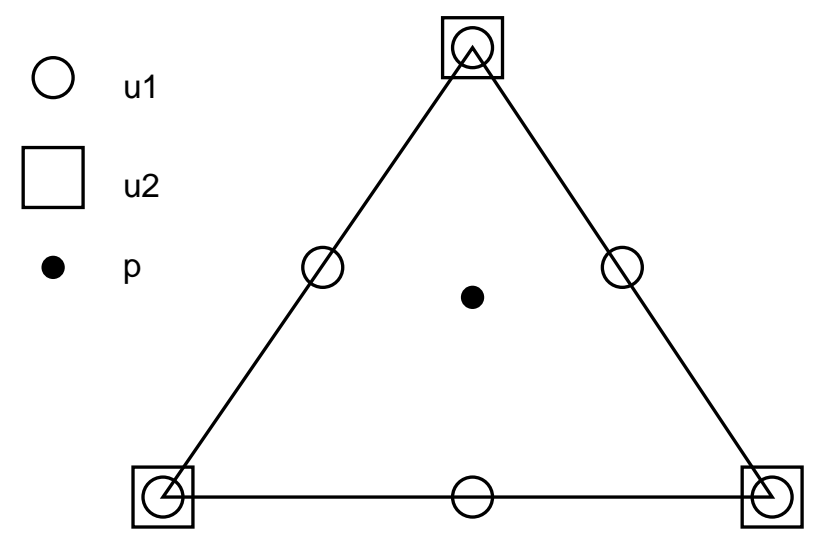

Fig. 2. The finite element $\mathbb{P}_{2} / \mathbb{P}_{1} / \mathbb{P}_{0}$. The degrees of freedom for each function $u_{1}, u_{2}$ and $p$ are indicated with a specific symbol.

\subsection{Analysis of the discrete problem}

The aim of this section is to analyze the discrete problem (22)-(23). It will be proved that it admits at least one solution. We start with the following technical, but fundamental result:

Lemma 1. The mapping

$$
\|.\|_{\mathrm{x}_{2}}: \quad \begin{aligned}
& \boldsymbol{H}_{\boldsymbol{h}, \boldsymbol{b}} \\
& \boldsymbol{v}_{h}=\left(v_{h, 1}, v_{h, 2}\right) \longmapsto \mathbb{R}
\end{aligned}\left\|\boldsymbol{v}_{h}\right\|_{\mathrm{x}_{2}}=\left\|\partial_{x_{2}} v_{h, 1}\right\|_{0, \Omega},
$$

defines a norm on $\boldsymbol{H}_{\boldsymbol{h}, \boldsymbol{b}}$.

Proof. The only property to check is that $\left\|\boldsymbol{v}_{h}\right\|_{\mathrm{x}_{2}}=0$ implies $\boldsymbol{v}_{h}=\mathbf{0}$ in $\Omega$. The other properties arise directly from the fact that $\|.\|_{0, \Omega}$ is a norm on $\mathcal{L}^{2}(\Omega)$. Let us consider $\boldsymbol{v}_{h} \in \boldsymbol{H}_{\boldsymbol{h}, \boldsymbol{b}}$ such that $\left\|\boldsymbol{v}_{h}\right\|_{\mathrm{x}_{2}}=0$. The Poincaré inequality (see [14])

$$
\left\|v_{h, 1}\right\|_{0, \Omega} \leq C(\Omega)\left\|\partial_{x_{2}} v_{h, 1}\right\|_{0, \Omega},
$$

implies that $v_{h, 1}=0$ in $\Omega$. As a result, $v_{h, 2}$ satisfies

$$
\forall q_{h} \in \Pi_{h},\left(q_{h}, \partial_{x_{2}} v_{h, 2}\right)_{\Omega}=0
$$

Let us additionally remark that the function $v_{h, 2}$ can be written as follows on each triangle $K_{i}$ of the mesh:

$$
\left.v_{h, 2}\right|_{K_{i}}\left(x_{1}, x_{2}\right)=\alpha_{i}+\beta_{i} x_{1}+\gamma_{i} x_{2} .
$$

For two given elements $K_{i}$ and $K_{j}$ of the mesh, the function

$$
\tilde{q}_{h}=\frac{1}{\left|K_{i}\right|} \mathbb{1}_{K_{i}}-\frac{1}{\left|K_{j}\right|} \mathbb{1}_{K_{j}}
$$

belongs to $\Pi_{h}$, and then, using (26) we easily see that

$$
\gamma_{i}=\gamma_{j}
$$


Let $\mathcal{D}=\left\{\left(x_{1}^{0}, x_{2}\right) \mid x_{2} \in \mathbb{R}\right\}$ be a vertical line, for any arbitrary $x_{1}^{0}$ such that $\mathcal{D} \cap \Omega \neq \emptyset$. This line intersects the mesh in a sequence of adjacent triangles $\left(K_{i}\right)_{i \in\{0, \ldots, n\}}$. The property (29) and the continuity of $v_{h, 2}$ imply that:

$$
\forall i \in\{0, \ldots, n\}, \alpha_{i}=\alpha_{0}, \beta_{i}=\beta_{0}, \gamma_{i}=\gamma_{0} .
$$

The boundary conditions that satisfies $v_{h, 2}$ are then such that: $\alpha_{0}=\beta_{0}=\gamma_{0}=0$. Since the same argument may be used for every (or almost every) $x_{1}^{0}$ such that $\mathcal{D} \cap \Omega \neq \emptyset$, then $\boldsymbol{v}_{h}=\mathbf{0}$ in $\Omega$.

Remark. A consequence of this lemma is that $\left(\boldsymbol{H}_{\boldsymbol{h}, \boldsymbol{b}},(\cdot, \cdot)_{\mathrm{x}_{2}}\right)$ is a Hilbert space with the scalar product : $\left(\boldsymbol{u}_{h}, \boldsymbol{v}_{h}\right)_{\mathrm{x}_{2}}=\left(\partial_{x_{2}} u_{h, 1}, \partial_{x_{2}} v_{h, 1}\right)_{\Omega}$.

Lemma 2. For $\lambda=0$, the problem (22) admits at least one solution $\boldsymbol{u}_{h} \in \boldsymbol{H}_{\boldsymbol{h}, \boldsymbol{b}}$.

Proof. We follow an approach similar to the one presented in [19] for the full Navier-Stokes equations. In $\left(\boldsymbol{H}_{\boldsymbol{h}, \boldsymbol{b}},(\cdot, \cdot)_{\mathrm{x}_{2}}\right)$, finite dimensional Hilbert space, we introduce the mapping $f$ from $\boldsymbol{H}_{\boldsymbol{h}, \boldsymbol{b}}$ into itself as follows. For $\boldsymbol{v}_{h} \in \boldsymbol{H}_{\boldsymbol{h}, \boldsymbol{b}}, f\left(\boldsymbol{v}_{h}\right)$ is the unique vector such that:

$$
\forall \boldsymbol{w}_{h} \in \boldsymbol{H}_{\boldsymbol{h}, \boldsymbol{b}}, \quad\left(f\left(\boldsymbol{v}_{h}\right), \boldsymbol{w}_{h}\right)_{\mathrm{x}_{2}}=c_{\mathrm{a}}\left(\boldsymbol{v}_{h}, \boldsymbol{v}_{h}, \boldsymbol{w}_{h}\right)+a\left(\boldsymbol{v}_{h}, \boldsymbol{w}_{h}\right)-\left(\boldsymbol{g}, \boldsymbol{w}_{h}\right)_{\Omega} .
$$

It is easy to check that $f$ is a continuous mapping. Now, for $\lambda=0$, $a$ satisfies

$$
\forall \boldsymbol{v}_{h} \in \boldsymbol{H}_{\boldsymbol{h}, \boldsymbol{b}}, \quad a\left(\boldsymbol{v}_{h}, \boldsymbol{v}_{h}\right)=\frac{1}{\operatorname{Re}}\left\|\boldsymbol{v}_{h}\right\|_{\mathrm{x}_{2}}^{2},
$$

and, using the Cauchy-Schwarz inequality and the equivalence of norms in a finite dimensional space there exists a positive constant $C_{1, h}$ such that:

$$
\forall \boldsymbol{v}_{h} \in \boldsymbol{H}_{\boldsymbol{h}, \boldsymbol{b}}, \quad\left|\left(\boldsymbol{g}, \boldsymbol{v}_{h}\right)_{\Omega}\right| \leq C_{1, h}\|\boldsymbol{g}\|_{0, \Omega}\left\|\boldsymbol{v}_{h}\right\|_{\mathrm{x}_{2}} .
$$

Using the previous results, $f$ satisfies

$$
\begin{aligned}
\left(f\left(\boldsymbol{v}_{h}\right), \boldsymbol{v}_{h}\right)_{\mathrm{x} 2} & =c_{\mathrm{a}}\left(\boldsymbol{v}_{h}, \boldsymbol{v}_{h}, \boldsymbol{v}_{h}\right)+a\left(\boldsymbol{v}_{h}, \boldsymbol{v}_{h}\right)-\left(\boldsymbol{g}, \boldsymbol{v}_{h}\right)_{\Omega} \\
& =a\left(\boldsymbol{v}_{h}, \boldsymbol{v}_{h}\right)-\left(\boldsymbol{g}, \boldsymbol{v}_{h}\right)_{\Omega} \\
& \geq \frac{1}{\operatorname{Re}}\left\|\boldsymbol{v}_{h}\right\|_{\mathrm{x}_{2}}^{2}-C_{1, h}\|\boldsymbol{g}\|_{0, \Omega}\left\|\boldsymbol{v}_{h}\right\|_{\mathrm{x}_{2}} \\
& \geq\left\|\boldsymbol{v}_{h}\right\|_{\mathrm{x}_{2}}\left(\frac{1}{\operatorname{Re}}\left\|\boldsymbol{v}_{h}\right\|_{\mathrm{x}_{2}}-C_{1, h}\|\boldsymbol{g}\|_{0, \Omega}\right) .
\end{aligned}
$$

If we choose $k>\operatorname{Re} C_{1, h}\|\boldsymbol{g}\|_{0, \Omega}$, then for $\left\|\boldsymbol{v}_{h}\right\|_{\mathrm{x}_{2}}=k,\left(f\left(\boldsymbol{v}_{h}\right), \boldsymbol{v}_{h}\right)_{\mathrm{x}_{2}}>0$. As a result, the lemma 1.4 p.164 in [19] ensures the existence of a solution $\boldsymbol{u}_{h}$ of the equation $f\left(\boldsymbol{u}_{h}\right)=0$, in other words, a solution of the discrete problem (22).

Remark. For the inequality (33), a better majoration can be given in the case $g_{2}=0$. Indeed, from the Cauchy-Schwarz inequality and the Poincaré inequality, we have:

$$
\forall \boldsymbol{v}_{h} \in \boldsymbol{H}_{\boldsymbol{h}, \boldsymbol{b}}, \quad\left|\left(\boldsymbol{g}, \boldsymbol{v}_{h}\right)_{\Omega}\right| \leq C(\Omega)\left\|g_{1}\right\|_{0, \Omega}\left\|\boldsymbol{v}_{h}\right\|_{\mathrm{x}_{2}} .
$$

Hence, if we define $k_{0}=\left(\operatorname{Re} C(\Omega)\left\|g_{1}\right\|_{0, \Omega}\right)$, the Lemma 1.4 in [19] also ensures that $\left\|\boldsymbol{u}_{h}\right\|_{\mathrm{x}_{2}} \leq$ $k_{0}$, thus $\left\|u_{h, 1}\right\|_{0, \Omega} \leq C(\Omega) k_{0}$, using again the Poincaré inequality. In other words, the set $\left\{\left\|u_{h, 1}\right\|_{0, \Omega}\right\}_{(h>0)}$ is bounded.

For the problem (23), we now have the following lemma: 
Lemma 3. The pair $\mathbb{P}_{2} / \mathbb{P}_{1} / \mathbb{P}_{0}$ is inf-sup stable, i.e., there exists a constant $\beta>0$, independent of $h$, such that:

$$
\inf _{q_{h} \in \Pi_{h}} \sup _{\boldsymbol{v}_{h} \in \boldsymbol{H}_{\boldsymbol{h}}} \frac{b\left(q_{h}, \boldsymbol{v}_{h}\right)}{\left\|\boldsymbol{v}_{h}\right\|_{\boldsymbol{H}(\Omega)}|| q_{h} \|_{0, \Omega}} \geq \beta .
$$

Then, for a given $\boldsymbol{u}_{h}$, the problem (23) admits one unique solution $p_{h} \in \Pi_{h}$.

Proof. In [18] it is proved that

$$
\inf _{q_{h} \in \Pi_{h}} \sup _{\boldsymbol{v}_{h} \in \boldsymbol{H}_{\boldsymbol{h}}} \frac{b\left(q_{h}, \boldsymbol{v}_{h}\right)}{\left\|\boldsymbol{v}_{h}\right\|_{1, \Omega}\left\|q_{h}\right\|_{0, \Omega}} \geq \beta,
$$

and the result arises from (9).

Collecting the previous results, we can state the main theorem of this section:

Theorem 1. The problem (22)-(23) admits at least one solution $\left(\boldsymbol{u}_{h}, p_{h}\right)$. Furthermore, in the case of $g_{2}=0$, the set $\left\{\left\|u_{h, 1}\right\|_{0, \Omega}\right\}_{(h>0)}$ is bounded by $\left(C(\Omega)^{2} \operatorname{Re}\left\|g_{1}\right\|_{0, \Omega}\right)$, where $C(\Omega)$ is the constant from the Poincaré inequality.

\section{Remark :}

(1) For the Taylor-Hood element and the Mini element, the inf-sup condition is also valid, which ensures that the discrete problem (23) has a unique solution for a given $\boldsymbol{u}_{h}$. Nevertheless, for these elements, and $\lambda=0$, the problem (22) might have no solution. Indeed, Lemma 2 might not be valid since for these elements, $\|.\|_{x_{2}}$ might not be a norm on $\boldsymbol{H}_{\boldsymbol{h}, \boldsymbol{b}}$ (note that the specific properties of the $\mathbb{P}_{2} / \mathbb{P}_{1} / \mathbb{P}_{0}$ element have been used in the proof of Lemma 1). This has been confirmed by the numerical experiments that fail for these elements.

(2) For $\lambda>0$, the lemmas and theorem above are of course still valid and the discrete problem still admits a solution.

(3) Note that no majoration of the transverse velocity $u_{h, 2}$ has been provided. This is due to the very particular nature of the RNS/P equations, that allow a weak control on this variable.

\subsection{Description of the algorithm of resolution}

For numerical simulations, boundary conditions that are not homogeneous have been considered. More precisely, a Poiseuille profile has been chosen for the entry:

$$
u_{1}^{0}\left(x_{2}\right)=4\left(1-x_{2}\right) x_{2} .
$$

As the problem is non-linear, the Newton method has been used. At each step of the Newton loop, the linearized discrete problem is solved using a multi-frontal Gauss LU factorization (cf. [7]) implemented in the package UMFPACK ${ }^{1}$. The complete scheme of the numerical resolution is given in Fig. 3.

\footnotetext{
${ }^{1}$ http://www.netlib.org/linalg
} 
The first numerical parameter is $\lambda$, which is the coefficient for the least-squares term $a_{\lambda}(\cdot, \cdot)$. It has been chosen for the numerical experiments as a constant and not as a scalar function. The two other parameters are $\left(n_{\mathrm{Re}}, n_{\mathrm{N}}\right)$ which are the number of steps in each of the two loops, respectively the loop 1 on the Reynolds number and the loop 2 which is the Newton loop (Fig. 3). The convergence has been measured through computation of:

$$
\left\|\left(\boldsymbol{d u}_{h}, d p_{h}\right)\right\|_{\max }=\max _{\mathcal{T}_{h}}\left\|\left(\boldsymbol{d} \boldsymbol{u}_{h}, d p_{h}\right)\right\|_{2}
$$

which value is usually of the order of $10^{-7}$ at the end of the simulation. All the numerical results have been obtained using FreeFEM++ software [10].

\section{Numerical results and discussion}

The problem consists of computing the fluid flow in a constricted pipe, a type of geometry which corresponds to a great variety of situations: flow in a Venturi pipe [20], in a collapsible tube [3], in a stenosis [2], in the vocal folds [16] or in the human pharynx [17], etc. The geometry can either be symmetric (for instance in a stenosis) or asymmetrical (for instance in the human pharynx or at the base of the tongue). Here, we have considered the asymmetrical problem. The characteristics of one representative simplified geometry are given Fig. 4: it is a straight pipe which is constricted because of a bump in the upper border. The pertinent parameters for this type of problem are the width $\delta$ and the height $h_{b}$ of the bump, as well as the Reynolds number Re.

The simulations have been carried out for three types of geometries:

- (geometry 1) A long pipe with a slightly curved upper wall $\left(\delta=5, h_{b}=0.2\right)$. It corresponds to an ideal case in which the assumptions of validity of the RNS/P equations should be encountered.

- (geometry 2) A pipe with a small obstacle $\left(\delta=0.1, h_{b}=0.2\right)$, a case described in [12]. It permits to test the method in a more realistic situation, with separation of the flow above a given Reynolds number.

- (geometry 3) A severe constriction $\left(\delta=0.5, h_{b}=0.5\right)$, a case described in [13]. The interest is to test the method and the model itself in a situation corresponding to the limit of validity of the RNS/P equations.

The meshes for each case are depicted Fig. 5. The range for the Reynolds number Re is $1-1000$ for the geometry 1, $1-500$ for the geometry 2 and $1-100$ for the geometry 3 . In a first set of experiments, the value for $\lambda$ has been fixed to 0 . The other numerical parameters $\left(n_{\operatorname{Re}}, n_{\mathrm{N}}\right)$ vary for each simulation and are therefore indicated each time. For comparison, the complete Navier-Stokes equations have been solved, on the same geometry, with the same mesh and the same parameters. Taylor-Hood elements have been used for this purpose. The pressure drop $\Delta P$ between the inlet and the outlet, which is an output of the simulations, has been compared. Moreover, the force $\boldsymbol{F}_{\text {sup }}$ exerted by the fluid on the upper wall has been computed since it is of particular interest in the case of fluid-structure interaction. This force is defined as 


$$
\boldsymbol{F}_{\text {sup }}=\int_{\Gamma_{\text {sup }}} \boldsymbol{\sigma}_{f} \boldsymbol{n} \mathrm{d} \Gamma,
$$

where $\Gamma_{\text {sup }}$ is the upper part of the boundary, $\boldsymbol{\sigma}_{f}$ is the tensor of fluid constraints and $\boldsymbol{n}$ is the inner unit vector normal to the boundary. It is of interest to decompose $\boldsymbol{F}_{\text {sup }}$ as:

$$
\boldsymbol{F}_{\text {sup }}=\boldsymbol{F}_{\text {sup }}^{p}+\frac{1}{\operatorname{Re}} \boldsymbol{F}_{\text {sup }}^{\tau}
$$

where $\boldsymbol{F}_{\text {sup }}^{p}$ is the contribution of the pressure:

$$
\boldsymbol{F}_{\mathrm{sup}}^{p}=\int_{\Gamma_{\mathrm{sup}}}(-p \boldsymbol{n}) \mathrm{d} \Gamma,
$$

and $\boldsymbol{F}_{\text {sup }}^{\tau}$ is the contribution of the shear stress:

$$
\boldsymbol{F}_{\text {sup }}^{\tau}=\int_{\Gamma_{\text {sup }}}\left(\nabla \boldsymbol{u}+\nabla \boldsymbol{u}^{\mathrm{T}}\right) \boldsymbol{n} \mathrm{d} \Gamma .
$$

In practice, for incompressible flows, the contribution from the shear stress is negligible with respect to the contribution from the pressure (see e.g. [6]). The results of the computations and of the comparisons (pressure drop $\Delta P$ and quadratic norms of $\boldsymbol{F}_{\text {sup }}, \boldsymbol{F}_{\text {sup }}^{p}, \boldsymbol{F}_{\text {sup }}^{\tau}$ ) are presented in Table 1.

For the geometry 1 and for a Reynolds number of 1000, the pressure distribution $p$ and the horizontal velocity $u_{1}$ are depicted Fig. 6. The predictions of $\Delta P$ and $\boldsymbol{F}_{\text {sup }}$ are in good adequation with those from the Navier-Stokes simulations, taken as a reference. The maximal error

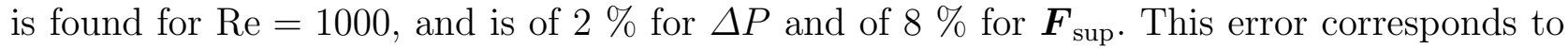
the quantity

$$
\frac{\left|\Delta P_{\mathrm{NS}}-\Delta P_{\mathrm{RNS} / \mathrm{P}}\right|}{\Delta P_{\mathrm{NS}}}
$$

where NS indicates the prediction from Navier-Stokes simulations and RNS/P the prediction from RNS/P simulations (the same computation is done for $\boldsymbol{F}_{\text {sup }}$ ). As a result, the simulations for this geometry have permitted to validate the numerical method.

For the geometry 2 and $\mathrm{Re}=100$, the pressure distribution $p$ and the horizontal velocity $u_{1}$ are depicted in Fig. 7. For $\operatorname{Re}=1$, the prediction of $\Delta P$ and $\boldsymbol{F}_{\text {sup }}$ corresponds to the prediction from Navier-Stokes simulations, with errors of $6 \%$ and $1.5 \%$, respectively. When the Reynolds number Re is 100, and the convection such that recirculation effects are observed behind the obstacle in Navier-Stokes simulations, the prediction of $\Delta P$ and $\boldsymbol{F}_{\text {sup }}$ remains satisfying (errors of $9 \%$ and $2 \%$ respectively), though the RNS/P equations are in principle not adapted for the simulation of recirculation effects, because of the assumption $\partial_{x_{2}} p=0$. The reason is that in this case, the effects of recirculation are weak. Note however that it affects the values of the velocity and therefore the value of $\boldsymbol{F}_{\text {sup }}^{\tau}$, which is different between RNS/P and Navier-Stokes simulations (error of $37.5 \%$ ). For a Reynolds number Re of 500, the recirculation is stronger. This does not prevent the RNS/P simulation to converge but the results are quite different 
from those of the Navier-Stokes simulation: an error of $24 \%$ for $\Delta P$ and an error of $36 \%$ for $\boldsymbol{F}_{\text {sup }}$.

For the geometry 3 and $\mathrm{Re}=1, p$ and $u_{1}$ are depicted Fig. 8. The adequation between RNS/P and Navier-Stokes simulations is still satisfying for Re $=1$, with an error of $13.5 \%$ for $\Delta P$ and an error of $8 \%$ for $\boldsymbol{F}_{\text {sup. }}$. This is slightly higher than in the precedent cases, but this fact is somehow expected since for this kind of geometry the RNS/P equations are in their limit of validity. Due to the recirculation effects, the error is more important when Re is increased to 50 or 100 , and is up to $23 \%$ for $\Delta P$ and $21 \%$ for $\boldsymbol{F}_{\text {sup }}$ (for $\operatorname{Re}=100$ ).

The influence of the least-squares term $a_{\lambda}(\cdot, \cdot)$ has finally been assessed. Simulations have been carried out with $\lambda=1$ for the geometry 2 . For $\mathrm{Re} \leq 100$, the results are very close to the ones obtained with $\lambda=0$, so that the difference with the Navier-Stokes simulations is nearly the same. Concerning the prediction of the shear stress component $\boldsymbol{F}_{\text {sup }}^{\tau}$ at $\operatorname{Re}=100$, the results are better with $\lambda=1$, as the error is of $15 \%$ instead of the $37.5 \%$ mentioned previously for $\lambda=0$. For $\operatorname{Re}>100$, some results are presented in Table 2 . It appears clearly that the leastsquares term improves the performance of prediction. Concerning $\Delta P$, the error is of $7.5 \%$ for $\lambda=1$ instead of $14 \%$ for $\lambda=0$ when $\operatorname{Re}=200$ (respectively, $6 \%$ and $24 \%$ when $\operatorname{Re}=500$ ). For $\boldsymbol{F}_{\text {sup }}$, the error decreases from $5 \%$ to $2 \%$ when $\lambda$ changes from 0 to 1 , when $\operatorname{Re}=200$ (respectively from $36 \%$ to $15 \%$ when $\mathrm{Re}=500$ ). Indeed, when the Reynolds number is high and the convection effects become predominant in the fluid, the least-square term allows an ad-hoc reproduction of the recirculation behind the obstacle, though underestimated if compared to those observed in the Navier-Stokes simulations. This explains the better estimation of $\boldsymbol{F}_{\text {sup }}^{\tau}$ (see Table 2) and $\boldsymbol{F}_{\text {sup }}$ in particular.

\section{Concluding remarks}

A finite element method to solve the Reduced Navier-Stokes/Prandtl (RNS/P) equations has been described and tested. The discretization is based on an element originally defined in [18] for the Stokes equations. With this element, called $\mathbb{P}_{2} / \mathbb{P}_{1} / \mathbb{P}_{0}$, the velocity is approximated with continuous piecewise quadratic and linear functions, while the pressure is approximated with a piecewise constant function. This element, as Taylor-Hood and the Mini element, verifies the inf-sup condition for the RNS/P equations. However, the subproblem of computation of the discrete velocity $\boldsymbol{u}_{h}$ might be ill-posed for the Taylor-Hood and the Mini element, which systematically leaded to failures in the numerical experiments. In opposite, we show that with the proposed $\mathbb{P}_{2} / \mathbb{P}_{1} / \mathbb{P}_{0}$ element, this subproblem admits a solution. Furthermore, it was shown that the longitudinal component of $\boldsymbol{u}_{h}$ is bounded by a constant independent of $h$, the parameter of discretization.

Numerical experiments permitted to test the method for some particular geometries, and to show that the prediction of the pressure drop and of the constraints on the surrounding walls is comparable to the prediction from Navier-Stokes simulation. Moreover, the current method avoids limitations of the precedent finite differences method used for instance in [11], which lacks of adaptivity for some geometries and for coupling with other physical entities, such as a 


\begin{tabular}{|c|c||c|||c|c|c|}
\hline geometry & $\operatorname{Re}$ & $\Delta P$ & $\left\|\boldsymbol{F}_{\text {sup }}\right\|$ & $\left\|\boldsymbol{F}_{\text {sup }}^{p}\right\|$ & $\left\|\boldsymbol{F}_{\text {sup }}^{\tau}\right\|$ \\
\hline \hline & 1 & 215.4 & 2218.0 & 2216.8 & 96.6 \\
\hline$(1)$ & 100 & 2.16 & 20.80 & 20.79 & 96.6 \\
\hline & 500 & 0.46 & 3.51 & 3.51 & 97.6 \\
\hline & 1000 & 0.26 & 1.62 & 1.62 & 100.1 \\
\hline \hline & 1 & 40.98 & 105.14 & 103.03 & 21.92 \\
\hline$(2)$ & 100 & 0.42 & 1.03 & 0.99 & 21.87 \\
\hline & 500 & 0.10 & 0.17 & 0.17 & 20.81 \\
\hline \hline & 1 & 71.33 & 157.66 & 150.72 & 31.96 \\
\hline$(3)$ & 50 & 1.53 & 2.53 & 2.48 & 31.38 \\
\hline & 100 & 0.85 & 0.94 & 0.94 & 30.95 \\
\hline
\end{tabular}

\begin{tabular}{|c|c||c|||c|c|c|}
\hline geometry & Re & $\Delta P$ & $\left\|\boldsymbol{F}_{\text {sup }}\right\|$ & $\left\|\boldsymbol{F}_{\text {sup }}^{p}\right\|$ & $\left\|\boldsymbol{F}_{\text {sup }}^{\tau}\right\|$ \\
\hline \hline & 1 & 216.1 & 2174.8 & 2173.6 & 97.1 \\
\hline$(1)$ & 100 & 2.16 & 20.36 & 20.35 & 97.0 \\
\hline & 500 & 0.46 & 3.38 & 3.37 & 98.0 \\
\hline & 1000 & 0.25 & 1.51 & 1.50 & 100.3 \\
\hline \hline & 1 & 43.52 & 106.66 & 105.66 & 18.55 \\
\hline$(2)$ & 100 & 0.46 & 1.01 & 1.00 & 15.91 \\
\hline & 500 & 0.13 & 0.13 & 0.12 & 9.41 \\
\hline \hline & 1 & 82.47 & 171.20 & 165.16 & 26.58 \\
\hline$(3)$ & 50 & 1.85 & 2.92 & 2.90 & 24.35 \\
\hline & 100 & 1.10 & 1.19 & 1.19 & 21.16 \\
\hline
\end{tabular}

Table 1. Results of the computations for the RNS/P equations (left) and the full Navier-Stokes equations (right). The pressure difference and the force on the superior wall are indicated.

\begin{tabular}{|c||c||c|c|c|}
\hline equations & $\Delta P$ & $\left\|\boldsymbol{F}_{\text {sup }}\right\|$ & $\left\|\boldsymbol{F}_{\text {sup }}^{p}\right\| \mid\left\|\boldsymbol{F}_{\text {sup }}^{\tau}\right\|$ \\
\hline \hline Navier-Stokes & 0.25 & 0.46 & 0.45 & 13.79 \\
\hline RNS/P $(\lambda=0)$ & 0.21 & 0.48 & 0.48 & 21.56 \\
\hline $\operatorname{RNS} / \mathrm{P}(\lambda=1)$ & 0.23 & 0.45 & 0.45 & 16.61 \\
\hline
\end{tabular}

\begin{tabular}{|c||c||c|c|c|}
\hline equations & $\Delta P$ & $\left\|\boldsymbol{F}_{\text {sup }}\right\|$ & $\left\|\boldsymbol{F}_{\text {sup }}^{p}\right\|$ & $\left\|\boldsymbol{F}_{\text {sup }}^{\tau}\right\|$ \\
\hline \hline Navier-Stokes & 0.13 & 0.13 & 0.12 & 9.41 \\
\hline RNS/P $(\lambda=0)$ & 0.10 & 0.17 & 0.17 & 20.81 \\
\hline RNS/P $(\lambda=1)$ & 0.12 & 0.15 & 0.15 & 13.09 \\
\hline
\end{tabular}

Table 2. Influence of the parameter $\lambda$ for simulations with the geometry 2. The Reynolds number Re is 200 (left) and 500 (right).

moving wall. Also, a bidimensional problem has been chosen for the simplicity of the presentation, but the extension to three-dimensional geometries should be easily carried out. Even in the case of moderate recirculation effects (small obstacle in a duct for instance), the method still gives a correct approximation of the predicted variables, whereas in the finite difference context, the computation had to be stopped, and only a prediction of the pressure and of the velocity on the upstream part of the domain was provided [13]. In the case of strong recirculation, for instance in a severe constriction, both finite differences and finite elements are unsatisfying. However, in this case, the RNS/P equations are out of their domain of validity, and a Navier-Stokes solver should be used instead for an accurate simulation.

A detailed study of the influence of the least-squares term $a_{\lambda}$, as well as the extension of this finite element method to a coupled fluid/structure interaction problem and to threedimensional geometries will be the subject of future research.

\section{Acknowledgements}

The work of Gabriel Barrenechea has been partially supported by CONICYT through FONDECYT Project $N^{\circ} 1061032$ and FONDAP program in Applied Mathematics. The work of Franz Chouly has been funded by Alfa project and the Fundación Andes (Chile, grant C-14055). The authors would like to thank R. Rodríguez, C. Pérez, P.-Y. Lagrée, M. Fernández and J.-F. Gerbeau for many helpful discussions and comments. 


\section{References}

1. P. Azérad. Analyse des équations de Navier-Stokes en bassin peu profond et de l'équation de transport. PhD thesis, Université de Neuchâtel, Neuchâtel, Switzerland, 1996.

2. S.A. Berger and L-D Jou. Flows in stenotic vessels. Annual Review of Fluid Mechanics, 32:347-382, 2000.

3. P.W. Carpenter and T.J. Pedley, editors. Flow in Collapsible Tubes and Past Other Highly Compliant Boundaries, chapter 2. Flows in Deformable Tubes and Channels. Theoretical Models and Biological Applications (M. Heil and O.E. Jensen), pages 15-49. Kluwer, 2003.

4. A.J. Chorin and J.E Mardsen. A Mathematical Introduction to Fluid Mechanics - Third Edition. Springer-Verlag, 1993.

5. F. Chouly, A. Van Hirtum, P.-Y. Lagrée, J.-R. Paoli, X. Pelorson, and Y. Payan. Simulation of the retroglossal fluid-structure interaction during obstructive sleep apnea. Lecture Notes in Computer Science. LNCS 4072, pages $48-57,2006$.

6. F. Chouly, A. Van Hirtum, P.-Y. Lagrée, X. Pelorson, and Y. Payan. Numerical and experimental study of expiratory flow in the case of major upper airway obstructions with fluid-structure interaction. Journal of Fluids and Structures, 2007. to appear.

7. T.A. Davis and I.S. Duff. A combined unifrontal/multifrontal method for unsymmetric sparse matrices, 1999. Technical Report 20, CISE, University of Florida.

8. A. Ern and J.-L. Guermond. Theory and Practice of Finite Elements. Springer-Verlag, 2004.

9. P.M Gresho and R.L. Sani. Incompressible Flow and the Finite Element Method. John Wiley \& Sons, 2000.

10. F. Hecht, O. Pironneau, A. Le Hyaric, and K. Ohtsuka. Freefem++ 2.16-1 documentation, 2007. Webpage: http://www.freefem.org/ff++.

11. P.-Y. Lagrée, E. Berger, M. Deverge, C. Vilain, and A. Hirschberg. Characterization of the pressure drop in a 2D symmetrical pipe: some asymptotical, numerical and experimental comparisons. ZAMM - Journal of Applied Mathematics and Mechanics, 85(2):141-146, 2005.

12. P.-Y. Lagrée and S. Lorthois. The RNS/Prandtl equations and their link with other asymptotic descriptions: application to the wall shear stress scaling in a constricted pipe. International Journal of Engineering Science, 43:352-378, 2005.

13. P.-Y. Lagrée, A. Van Hirtum, and X. Pelorson. Asymmetrical effects in a 2D stenosis. European Journal of Mechanics B/Fluids, 26:83-92, 2007.

14. P.A. Raviart and J.M. Thomas. Introduction à l'Analyse Numérique des Équations aux Dérivées Partielles. Masson, 1992.

15. T.-A. Reyhner and Flügge-Lotz. The interaction of a shockwave with a laminar boundary layer. International Journal of Non-Linear Mechanics, 3:173-199, 1968.

16. R.-C. Scherer, D. Shinwari, K.-J. De Witt, C. Zhang, B.-R. Kucinschi, and A.-A. Afjeh. Intraglottal pressure profiles for a symmetric and oblique glottis with a divergence angle of 10 degrees. Journal of the Acoustical Society of America, 109:1616-1630, 2001.

17. B. Shome, L.-P. Wang, M.-H. Santare, A.-K. Prasad, A.-Z. Szeri, and D. Roberts. Modeling of airflow in the pharynx with application to sleep apnea. Journal of Biomechanical Engineering, 120:416-422, 1998.

18. R. Stenberg. Error analysis of some finite element methods for the Stokes problem. Mathematics of Computation, 54:495-508, 1990.

19. R. Temam. Navier-Stokes Equations. North-Holland, Elsevier, 1985.

20. S. Turek. Efficient Solvers for Incompressible Flow Problems. An Algorithmic and Computational Approach. Springer, 1999.

21. A. Van Hirtum, X. Pelorson, and P.-Y. Lagrée. In vitro validation of some flow assumptions for the prediction of the pressure distribution during obstructive sleep apnea. Medical \& Biological Engineering 6 Computing, 43:162-171, 2005.

22. O.E. Zienkiewicz, R.L. Taylor, and P. Nithiarasu. The Finite Element Method for Fluid Dynamics - Sixth Edition. Butterworth-Heinemann, 2005. 
Init:

- Read the simulation parameters and the mesh.

- Init the velocity and the pressure: $\left(\boldsymbol{u}_{h}, p_{h}\right)$.

- Init the Reynolds number Re.

Loop 1: Continuation strategy.

- Loop 2: Newton iteration.

- Solve the linearized problem:

Find $\left(\boldsymbol{d} \boldsymbol{u}_{h}, d p_{h}\right)$ such that

$$
\left\{\begin{array}{rr}
\forall \boldsymbol{v}_{h}, \quad & \\
c_{\mathrm{a}}\left(\boldsymbol{u}_{h}, \boldsymbol{d} \boldsymbol{u}_{h}, \boldsymbol{v}_{h}\right)+c_{\mathrm{a}}\left(\boldsymbol{d} \boldsymbol{u}_{h}, \boldsymbol{u}_{h}, \boldsymbol{v}_{h}\right)+ & \\
a\left(\boldsymbol{d} \boldsymbol{u}_{h}, \boldsymbol{v}_{h}\right)-\left(d p_{h}, \nabla \cdot \boldsymbol{v}_{h}\right)_{\Omega} & = \\
& \quad\left(\boldsymbol{g}, \boldsymbol{v}_{h}\right)_{\Omega}-c_{\mathrm{a}}\left(\boldsymbol{u}_{h}, \boldsymbol{u}_{h}, \boldsymbol{v}_{h}\right) \\
& -a\left(\boldsymbol{u}_{h}, \boldsymbol{v}_{h}\right)+\left(p_{h}, \nabla \cdot \boldsymbol{v}_{h}\right)_{\Omega}, \\
& \\
\forall q_{h}, \quad-\left(q_{h}, \nabla \cdot \boldsymbol{d} \boldsymbol{u}_{h}\right)_{\Omega}=\left(q_{h}, \nabla \cdot \boldsymbol{u}_{h}\right)_{\Omega} . &
\end{array}\right.
$$

- Update the velocity and the pressure:

\section{- End Loop 2.}

$$
\begin{aligned}
& \boldsymbol{u}_{h} \leftarrow \boldsymbol{u}_{h}+\boldsymbol{d} \boldsymbol{u}_{h} \\
& p_{h} \leftarrow p_{h}+d p_{h}
\end{aligned}
$$

- Increase the Reynolds number Re.

End Loop 1.

End.

Fig. 3. The complete algorithm of numerical solving of the RNS/P equations.

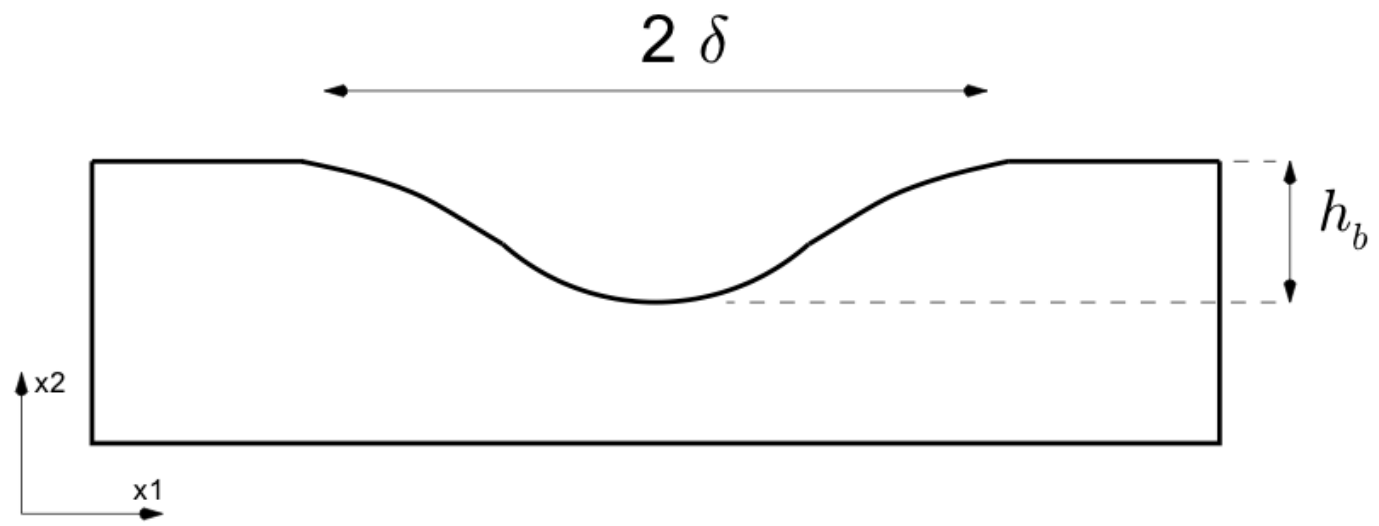

(a)

Fig. 4. The asymmetrical constricted pipe. 


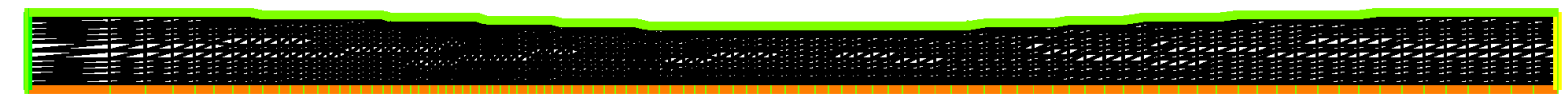

(a)

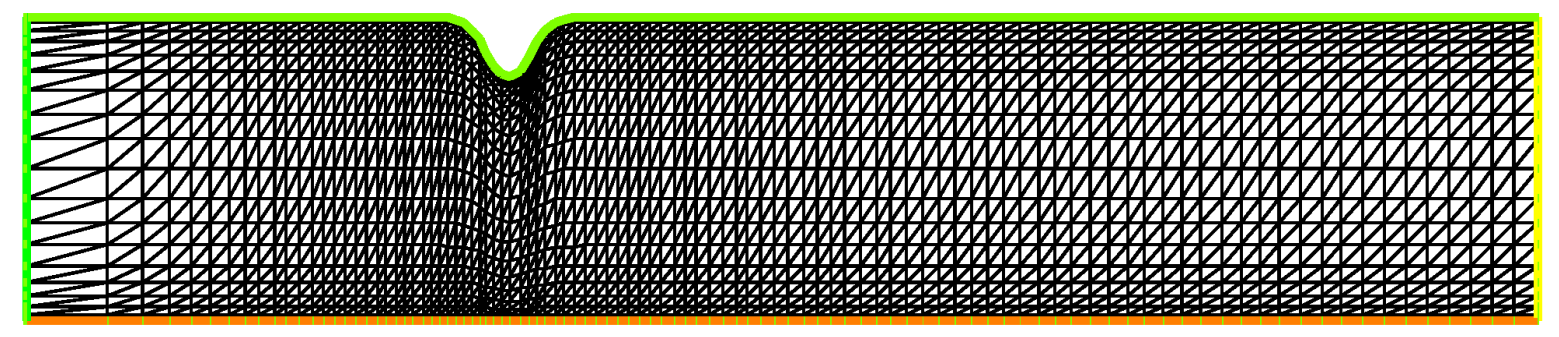

(b)

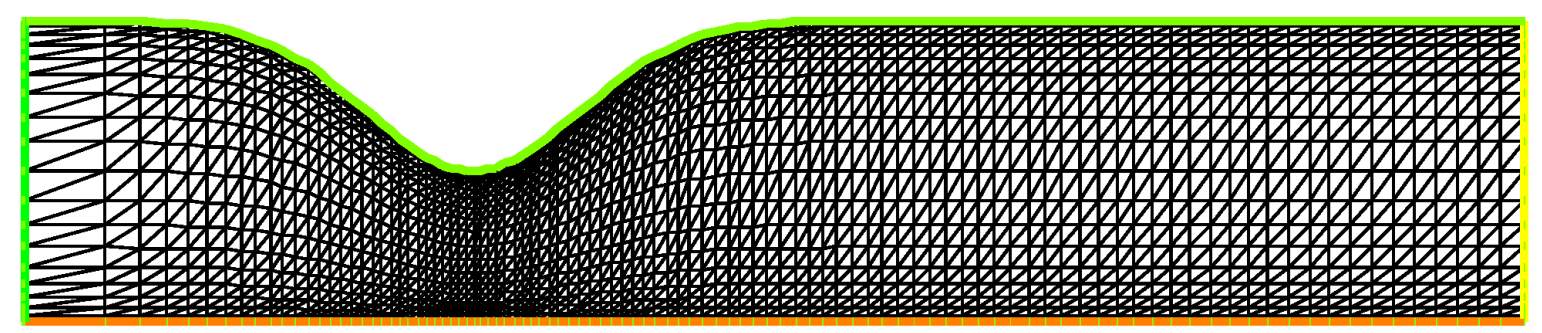

(c)

Fig. 5. The finite element meshes for the three fluid flow problems. 

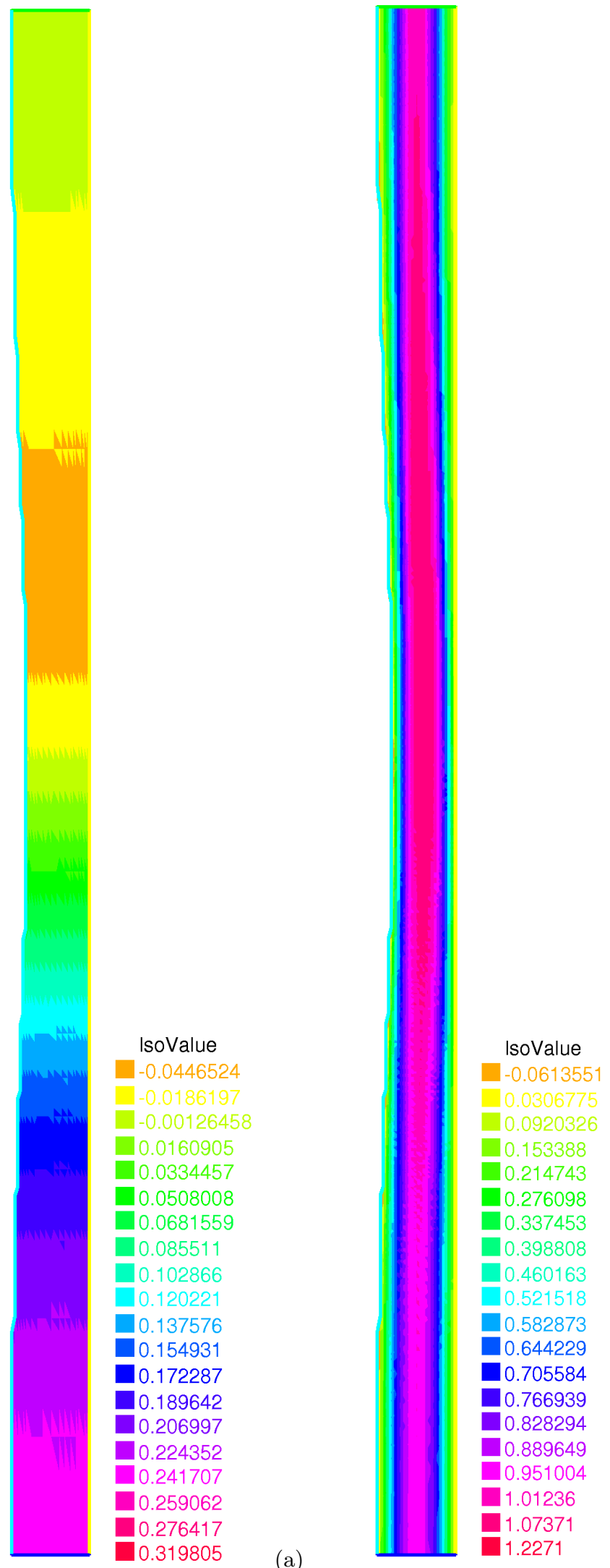

(a)

(b)

Fig. 6. Fluid flow in a constricted pipe. Geometry 1. Simulations with RNS $/ \mathrm{P}$ equations and $\mathbb{P}_{2} / \mathbb{P}_{1} / \mathbb{P}_{0}$ elements. $R e=1000 .\left(\lambda, n_{\mathrm{Re}}, n_{\mathrm{N}}\right)=(0,100,5)$. Are depicted: (a) the pressure distribution $p$, (b) the horizontal velocity $u_{1}$. 

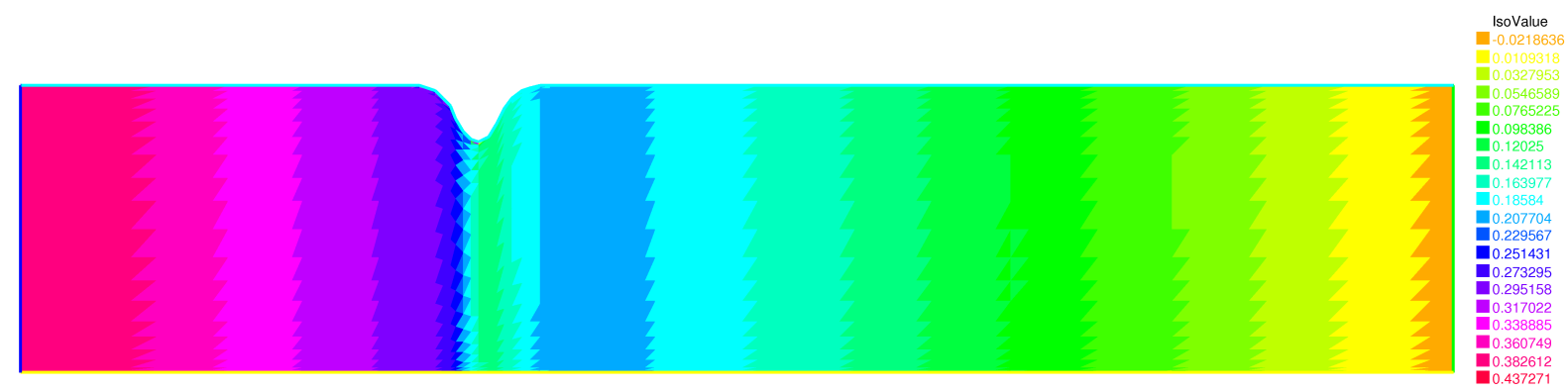

(a)

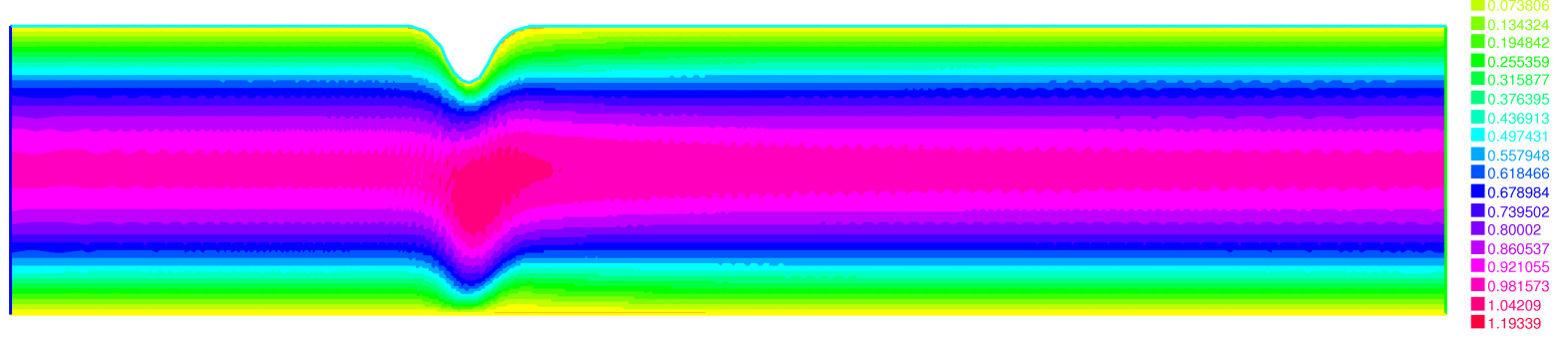

(b)

Fig. 7. Fluid flow in a constricted pipe. Geometry 2. Simulations with RNS/P equations and $\mathbb{P}_{2} / \mathbb{P}_{1} / \mathbb{P}_{0}$ elements. $R e=100 .\left(\lambda, n_{\mathrm{Re}}, n_{\mathrm{N}}\right)=(0,10,5)$. Are depicted: (a) the pressure distribution $p$. (b) the horizontal velocity $u_{1}$. 


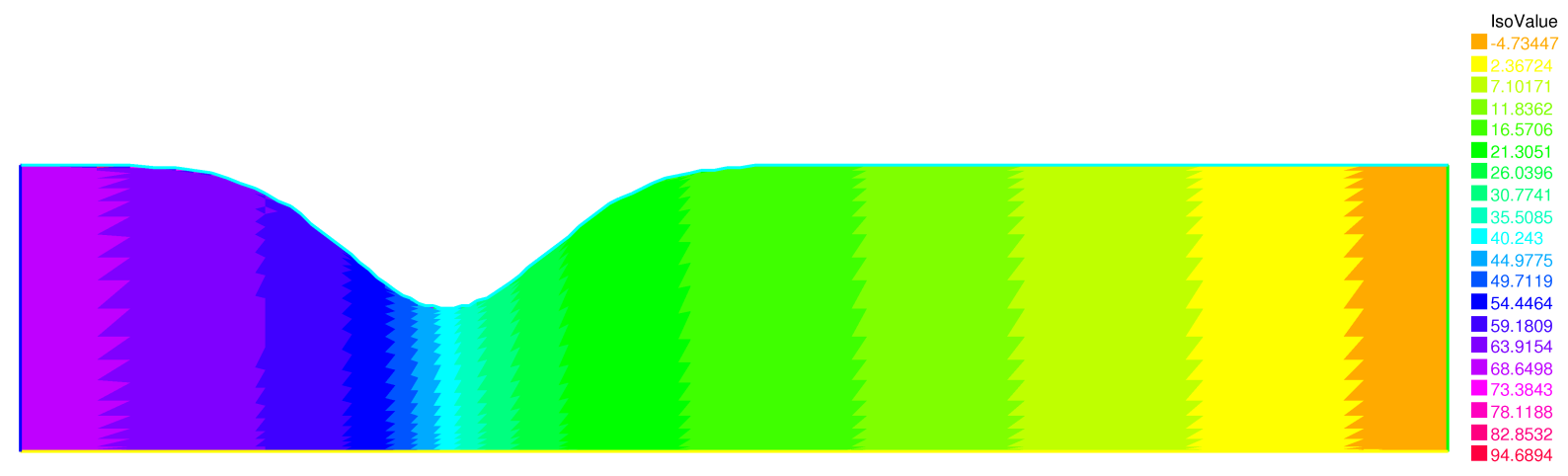

(a)

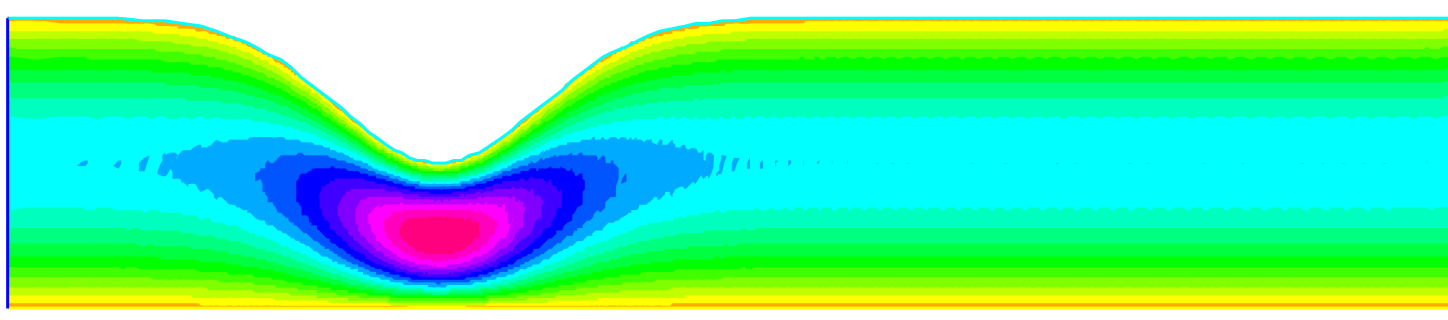

IsoValue

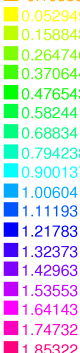

(b)

Fig. 8. Fluid flow in a constricted pipe. Geometry 3. Simulations with RNS $/ \mathrm{P}$ equations and $\mathbb{P}_{2} / \mathbb{P}_{1} / \mathbb{P}_{0}$ elements. Re $=1$. $\left(\lambda, n_{\mathrm{Re}}, n_{\mathrm{N}}\right)=(0,5,2)$. Are depicted: (a) the pressure distribution $p$. (b) the horizontal velocity $u_{1}$. 